

\title{
Postoperative MR Imaging of Spontaneous Transdural Spinal Cord Herniation: Expected Findings and Complications
}

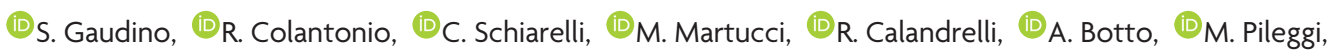 \\ (DE. Gangemi, (1) G. Maira, and (1)C. Colosimo
}

\begin{abstract}
BACKGROUND AND PURPOSE: Spontaneous transdural spinal cord herniation is no longer a rare cause of myelopathy. The high frequency of diagnoses has led to an increase in the number of surgical procedures. The purpose of this study was to describe the spectrum of postoperative MR imaging findings concerning spontaneous transdural spinal cord herniation and to provide a practical imaging approach for differentiating expected changes and complications after an operation.
\end{abstract}

MATERIALS AND METHODS: We retrospectively reviewed MR images from 12 patients surgically treated for spontaneous transdural spinal cord herniation. Surgery comprised either dural defect enlargement or duraplasty procedures. Postoperative follow-ups included at least 3 (early, intermediate, late) MR imaging studies. MR images were analyzed with respect to 3 spinal compartments: intradural intramedullary, intradural extramedullary, and extradural. The meaning and reliability of changes detected on MR images were related to their radiologic and clinical evolution with time.

RESULTS: Spinal cord realignment has been stable since the early study, whereas spinal cord signal and thickness evolved during the following scans. Most extramedullary and extradural changes gradually reduced in later MR images. Three patients treated with dural defect enlargements experienced the onset of new neurologic symptoms. In those patients, late MR images showed extradural fluid collection and the development of pial siderosis.

CONCLUSIONS: Our findings demonstrate the spectrum of postoperative imaging findings in spontaneous transdural spinal cord herniation. Spinal cord thickness and signal intensity continued to evolve with time; most extramedullary postsurgical changes became stable. Changes observed in later images may be suggestive of complications.

ABBREVIATIONS: DDE = dural defect enlargement; E-MRI = early MR imaging; I-MRI = intermediate MR imaging; JOA = Japanese Orthopedic Association; L-MRI = late MR imaging; STSCH = spontaneous transdural spinal cord herniation

$\mathbf{S}_{\mathrm{p}=\mathrm{p}}^{\mathrm{p}}$ pontaneous transdural spinal cord herniation (STSCH) is a rare, but potentially treatable cause of thoracic myelopathy that occurs in middle-aged adults, with a female preponderance. ${ }^{1}$ It is defined as the herniation or prolapse of the spinal cord through an anterior or anterolateral dural defect. Clinical findings are nonspecific, and patients are typically affected by a slowly progressive, Brown-Séquard syndrome or paraparesis. ${ }^{2}$ A variety

Received June 3, 2015; accepted after revision July 22.

From the Institute of Radiology (S.G., R. Colantonio, C.S., M.M., R. Calandrelli, A.B., M.P., E.G., C.C.) and Institute of Neurosurgery (G.M.), Catholic University of Rome, Rome, Italy.

Paper previously presented at: Annual Meeting of the Radiological Society of North America, November 25-30, 2012; Chicago, Illinois. Paper No. VSNR31-11.

Please address correspondence to Simona Gaudino, MD, Institute of Radiology, Catholic University, “A.Gemelli” Hospital. L.go A, Gemelli 1, 00168 Rome, Italy;

e-mail: simona.gaudino@rm.unicatt.it

三 Indicates article with supplemental on-line table.

http://dx.doi.org/10.3174/ajnr.A4537 of theories have been postulated to explain the pathogenesis of this entity, but the exact cause of dural defects remains unclear. ${ }^{1}$ Surgical treatment to restore a herniated cord to its normal intradural position is mandatory for maximum reversal of neurologic deficits. ${ }^{1}$ The primary techniques for treating STSCH are dural defect enlargement (DDE) and duraplasty. In recent years, the increased frequency of STSCH has led to more surgical treatments. These increases may be due to a growing awareness among clinicians and radiologists and to improvements in MR imaging quality. $^{2-4}$

A well-defined set of MR imaging diagnostic criteria has been established for STSCH${ }^{2,4-6}$; however, little has been said about postoperative MR imaging of changes in the spinal cord and adjacent structures. Less than half of the cases reported in the literature have described postoperative MR imaging features after reduction of spinal cord herniation ${ }^{1,4,7-10}$; none provided descriptions of a rational analysis; and in cases of complications, few have performed time-related assessments 
of the findings. The lack of a thorough knowledge of postoperative MR imaging findings and their evolution with time can lead to misinterpretations of normal postoperative changes and oversight of complications.

This study aimed to review the spectrum of postoperative MR imaging findings and provide a practical imaging approach for correctly differentiating normal postoperative findings and complications. This approach was based on our analysis of a case series of 12 patients who underwent surgery for STSCH and long-term follow-up with MR imaging and clinical assessment.

\section{MATERIALS AND METHODS Subjects}

We analyzed 17 consecutive patients who underwent surgery for STSCH by the same surgeon between 1985 and 2012. Inclusion criteria were radiologic diagnosis of STSCH surgically demonstrated and the availability of both preoperative and at least 3 postoperative MR imaging studies, with the last study conducted at least 1 year after the operation. Of the 17 patients considered, 12 (4 men and 8 women) were included in this analysis. Eleven patients were treated at our institution, and 5 of them have been previously reported in the literature. ${ }^{1}$ Clinical history, surgical outcomes, and radiographic findings were obtained for all patients. Medical records and MR imaging studies of the single patient not treated at our institution were collected by the surgeon during clinical follow-up. The mean age was 46 years (range, 29-61 years).

We have used 2 different surgical techniques: DDE for the first 3 patients and duraplasty for the other 9 patients. This difference is because after an initial experience with DDE, our surgeon preferred to repair the dural hole by using a dural patch, to reduce the risk of extradural fluid collection observed in patients whose dural defect was not repaired. ${ }^{1}$ All patients underwent pre- and postoperative spine MR imaging, and 3 patients also underwent brain MR imaging in late follow-up, due to neurologic deterioration. The median follow-up period was 7.8 years (range, 2-23 years). Due to the lack of a predefined MR imaging protocol for follow-up and in light of the retrospective nature of the study, the number and timing of postoperative MR imaging studies varied for each patient. In addition, the change of the clinical picture, with the onset of new neurologic symptoms many years after surgery, led to a very late imaging follow-up in 3 patients (from 9 to 17 years after the operation). However, in all cases, these studies consisted of at least 3 serial examinations of the spine conducted as follows: early MR imaging (E-MRI), conducted 7-10 days after the operation; intermediate MR imaging (I-MRI), conducted 90-120 days after the operation; and late MR imaging (LMRI), conducted $>12$ months after the operation. Postsurgical outcome was evaluated with the modified Japanese Orthopedic Association (JOA) score for thoracic myelopathy, an 11point scale measuring lower extremity motor function, lower extremity and trunk function, and bladder function. ${ }^{1,12}$

Informed consent was obtained from all patients, and the local institutional review board approved this study. All data were anonymized for review and publication.

\section{MR Imaging Protocol}

All postoperative MR imaging studies were performed with a $1.5 \mathrm{~T}$ scanner (Signa Horizon, Signa Infinity; GE Healthcare, Milwaukee, Wisconsin) and a 4- or 8-channel, dedicated spine coil. The MR imaging protocol consisted at least of the following sequences: sagittal, axial, and coronal T2WI fast spin-echo (TR, $>3000$ ms; TE, $>80 \mathrm{~ms}$; NEX, 3); and sagittal and axial T1WI fast spinecho (TR, <700 ms; TE, Minimum Full; NEX, 2-3) or spin-echo before and after intravenous administration of gadolinium $(0.1$ $\mathrm{mmol} / \mathrm{kg}$ ). Most MR imaging examinations also included fat-saturated T2WI and fat-saturated postcontrast T1WI; and 8 L-MRI also included gradient-echo T2* images (TR, 500-600 ms; TE, 20 $\mathrm{ms}$; flip angle, $20^{\circ}$; NEX, 3). Serial MR images were acquired at section thicknesses of 3-4 mm, with interimage gaps of $0.3-0.4$ $\mathrm{mm}$; the matrix size varied from $384 \times 256$ to $448 \times 320$. Steadystate free precession MR imaging sequences were obtained in the axial plane in all studies performed in 2003 or later. In addition, 3 patients underwent brain MR imaging studies that included gradient-echo $\mathrm{T} 2 *$ images.

\section{Image Analysis}

Pre- and postoperative MR images were retrospectively reviewed by 2 neuroradiologists (S.G. and C.S., with 10 and 2 years of experience, respectively); all determinations were made by consensus. For systematic image analysis, we used a compartmental approach, which followed the anatomic algorithm predicated on a myelographic interpretation ${ }^{13,14}$; consequently, the MR imaging findings were analyzed as follows:

1) In the intradural intramedullary compartment, we evaluated spinal cord realignment, dural adhesion of the spinal cord, abnormal thickness, abnormal signal intensity on the T2WI, and abnormal contrast enhancement of the spinal cord.

2) In the intradural extramedullary compartment, we evaluated the dural patch, the presence of siderosis on pial surfaces, intradural hemorrhage, and signs of CSF flow voids.

3) In the extradural compartment, we evaluated fluid collections.

A "spinal cord realignment" was defined as the repositioning of the spinal cord to its normal, intradural location, assessed on axial and sagittal T2WI. "Dural adhesion" was defined as a contact between the spinal cord and the ventral dura mater, without evidence of interposed CSF, assessed on axial T2WI and, when available, on steady-state free precession images. "Abnormal spinal cord thickness" was defined as a thinning or thickening of the spinal cord at the level of the operation, compared with the adjacent normal spinal cord, visible on T2WI and on at least 2 orthogonal planes. An "abnormal spinal cord signal intensity" on the T2WI was defined as a change in the spinal cord signal at the level of the operation, visible on at least 2 orthogonal planes (the conspicuity of the T2WI abnormality was further classified as either "faint" or "intense"). We also recorded abnormal spinal cord signal intensities on the T2WI that changed in longitudinal extension with time (classified as either "increased" or "decreased"). The dural patch was evaluated in terms of both the signal intensity on T2 and T1WI and the presence or absence of contrast enhancement. Extradural fluid collections were classified as either "anterior" or "posterior." All these MR imaging findings were 
evaluated across time to assess changes in terms of appearance, disappearance, progression, reduction, and stability.

\section{RESULTS}

\section{Postoperative MR Imaging Findings}

Intradural Intramedullary Compartment. In all patients, the EMRIs demonstrated that the spinal cord was realigned to the normal intradural location. No recurrence of spinal cord herniation was observed during the MR imaging follow-up. However, despite realignment, in 10 cases, a focal anterior dural adhesion was observed on the E-MRIs, and in 8 cases, it persisted on the LMRIs. The E-MRIs showed abnormal spinal cord thickness in all patients, but with different degrees of thinning at the site of surgery. Compared with the E-MRI, the I-MRIs showed that the spinal cord thickness had increased in 7 patients, remained stable in 3 patients, and decreased in 2 patients. Compared with the I-MRI, the L-MRIs showed that among the 7 spinal cords that had increased in thickness, 3 remained stable and 4 decreased; among the 3 spinal cords with a stable thickness, 1 remained stable and 2 decreased; and both spinal cords that had decreased thickness showed further thinning. Thus, at the end of the follow-up, 8 spinal cords showed progressive thinning and 4 remained stable.

On T2WI, the E-MRI showed abnormal spinal cord signal intensities in all patients at the level of the operation. During MR imaging follow-up, signal intensities increased in longitudinal extension in 4 patients: Two increases were observed between the E-MRI and I-MRI, and 2 were observed between the I-MRI and L-MRI. On the E-MRIs, the spinal cord signal intensity on T2WI was faint in 11 patients and intense in 1 patient; on the I-MRIs, it was faint in 7 and intense in 5 patients; and on the L-MRIs, it was faint in 3 and intense in 9 patients. Thus, 8 patients showed an overall modification in signal conspicuity at the end of the MR imaging follow-up. After administration of gadolinium, no patients showed abnormal contrast enhancement in the spinal cord.

Intradural Extramedullary Compartment. Among the 9 patients who underwent duraplasty, a dural patch was visible in 3 patients on E-MRIs and in 4 patients on I-MRIs and L-MRIs. The dural patch was a linear, T2 hyperintensity lying on the anterior surface of the dura, with variable signal intensity on T1WI (iso- to hypointensity). Dural patch contrast enhancement was observed in 1 case on the I-MRI. Posterior CSF flow artifacts were observed on the E-MRIs in 1 patient, on the I-MRIs in 8 patients, and on the L-MRIs in 9 patients. Intradural hemorrhage was observed on the E-MRIs in 2 patients at the level of the operation, but it completely resolved during the MR imaging follow-up. Spinal siderosis was observed in 1 patient on L-MRI.

Extradural Compartment. Posterior fluid collections were present in all patients on the E-MRI. They resolved in all except 2 on the I-MRIs, and they completely resolved in all patients on the L-MRIs. An anterior fluid collection adjacent to the site of previous herniation was present in 6 patients on the E-MRIs. In all cases, the anterior fluid collections were evident on preoperative MR images, and they began to show reductions on I-MRIs. In particular, they resolved on the I-MRIs in 3 patients and on the L-MRIs in the other 3 patients. An anterior extradural fluid col- lection with a cervicothoracic extension appeared on the L-MRI in 3 patients.

\section{Brain Findings}

Three patients had neurologic deterioration during follow-up (headache that worsened in the upright position, progressive sensorineural hearing loss, new-onset back pain). These patients required a brain MR imaging study. All 3 patients showed brain superficial siderosis.

\section{Postoperative Clinical Outcome}

The preoperative JOA score of all patients ranged from 4 to 9 (average, 6.5), whereas the postoperative JOA score at 1-year follow-up ranged from 5 to 10 (average 8), with an average recovery rate of $36 \%$. In particular, the postoperative score increased in 9 of 12 patients who showed a progressive improvement in spinal symptoms; in the remaining 3 patients, spinal symptoms and therefore the JOA score remained unchanged from the preoperative condition. There was no correlation between the postoperative JOA score and spinal cord changes. In fact, in our population, major spinal cord changes, in terms of cord thinning and T2WI signal extent, were present in 2 patients with an unvarying postoperative JOA score and in 3 patients with an increased postoperative JOA score. The 3 patients who had undergone DDE developed new clinical symptoms in the late clinical follow-up, including persistent headache that worsened in an upright position (2 cases), progressive sensorineural hearing loss (2 cases), and new-onset back pain (1 case).

\section{DISCUSSION}

STSCH is a rare, but treatable cause of slowly progressive thoracic myelopathy. It has been increasingly recognized and treated surgically in the past few years. Although great emphasis has been placed on the role of MR imaging in the diagnosis of STSCH, postoperative MR imaging findings have been exhaustively described in only a small number of reports, to our knowledge. Furthermore, no previous study has considered the time evolution in a series of MR imaging studies or attempted to interpret the clinical significance of postoperative changes and complications. In this study, we investigated MR images acquired after surgical treatment of STSCH in a series of MR imaging followups. Our aim was to differentiate normal or expected postoperative changes and complications on the basis of their evolution in time and the onset of new neurologic symptoms.

The major MR imaging findings of the present study (On-line Table) were the following: 1) Spinal cord changes, such as thickness and signal intensity on T2WI, could continue to evolve until L-MRIs; 2) most extramedullary postsurgical changes became stable after the I-MRI; and 3) changes that developed in the late phase that were associated with new neurologic symptoms could be considered a complication, like extradural anterior fluid collections or spinal superficial siderosis. These changes indicated a need for brain MR imaging because they could be associated with brain superficial siderosis.

The aim of STSCH surgery is to realign the spinal cord, to reposition it to its normal intradural location. ${ }^{15}$ This aim was well-accomplished in all patients on the basis of the E-MRIs; 

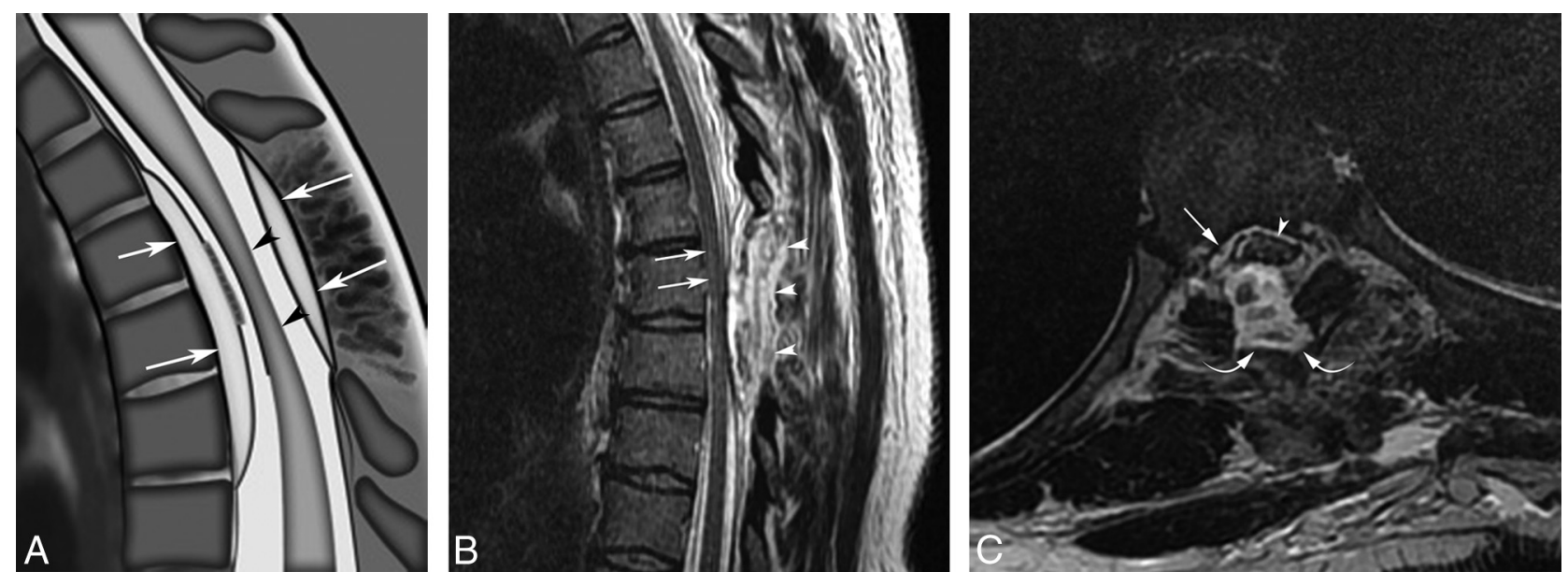

FIG 1. MR imaging findings after surgical treatment for STSCH. A, This drawing summarizes the expected MR imaging findings in early and intermediate MR imaging studies, including spinal cord realignment, reduced cord thickness at the site of herniation (black arrowheads), and anterior and posterior extradural fluid collections (white arrows). The thick dotted line along the anterior dura indicates the dural patch. $B$ and $C$, Early postoperative findings. B, Sagittal T2WI demonstrates intradural realignment, reduced thickness, and signal hyperintensity of the spinal cord (arrows) at the T6-T7 level (site of herniation) and a posterior fluid collection along the laminectomy (arrowheads). C, Axial T2WI shows the position of the spinal cord in the intradural location, an anterior adhesion between the cord and the dura (arrowhead), a thin anterior fluid collection (straight arrow), and a thick posterior fluid collection (curved arrows) with consequent total effacement of the posterior subarachnoid space.

moreover, later MRIs showed that all realignments remained stable. Thus, evaluations of realignments could be reliably based on the early postoperative MR imaging study (Fig $1 A$ ). However, despite correct realignments, we observed a focal contact between the ventral dura mater and the spinal cord in 10 patients on the E-MRIs. In our opinion, this finding might be due to the presence of a posterior extradural fluid collection, which may have caused a persistent, mild anterior displacement of the spinal cord toward the ventral dura (Fig 1B,-C). Although complete resorption of posterior extradural fluid collections occurred in all patients, dural adhesions disappeared in only 2 patients on the I-MRI and in 2 other patients at the end of the MR imaging follow-up. We speculate that adhesion disappearance was probably due to a reparative or reactive process.

Spinal cord changes at the site of the operation occurred in all patients, with a remarkable variability in terms of signal intensity on T2WI and thickness during the MR imaging follow-up. Several authors have investigated the meaning and evolution across time of alterations in spinal cord T2WI signal intensity after different types of spinal surgery. Many authors have hypothesized that intramedullary T2WI hyperintensities might represent a variety of histologic changes, including edema, ischemia, demyelination, gliosis, and microcavities. ${ }^{16}$ Yagi et al ${ }^{17}$ reported intramedullary signal-intensity changes up to 1 year after cervical laminoplasty, and Sarkar et $\mathrm{al}^{18}$ described the postoperative evolution of spinal cord T2WI, which changed in signal intensity and size, even after a long-term follow-up (mean duration, 28 months). Among our case series, some patients underwent a very long-term follow-up. In 2 patients, we detected T2WI signal intensity changes up to 10 years after the operation. In general, these signals tended to show progressive increases in both longitudinal extension and conspicuity; the conspicuity became intense in 9 patients at the end of follow-up. As recently proposed by Sarkar et al, ${ }^{18}$ we assumed that in the early postoperative phase, a faint T2WI hyperintensity was mainly related to the presence of edema. Accordingly, a progres- sive increase in conspicuity with time might be due to edema regression, which might unmask myelomalacia and/or gliosis. Therefore, in the setting of late postoperative spinal cord T2WI changes, the interpretation of these signal-intensity abnormalities should consider active pathogenic processes, such as alterations in venous circulation or arterial ischemia, which might indicate progression to myelomalacia and gliosis.

On E-MRIs, all patients showed decreases in spinal cord thickness at the site of the operation compared with adjacent areas of

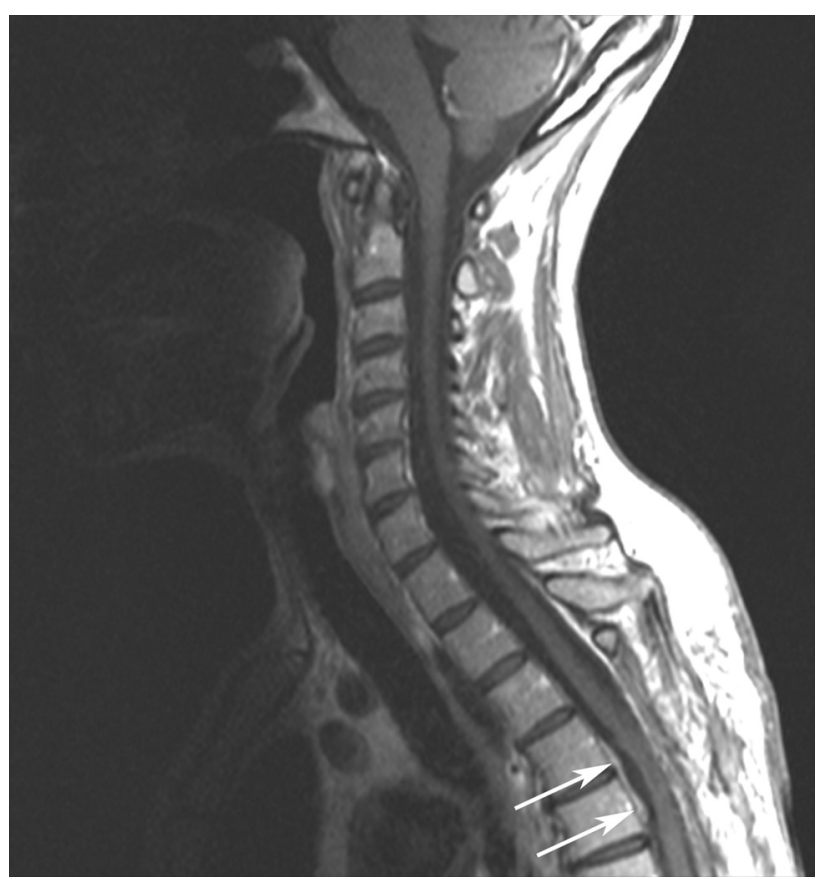

FIG 2. Dural patch showing contrast enhancement. Sagittal TTWI after intravenous administration of gadolinium shows the dural patch as a linear hyperintensity lying on the anterior surface of the dura (arrows) at the level of the operation (D4-D5 vertebral bodies). 
cord that appeared normal. On I-MRIs, 7 patients showed variable changes (increases or decreases in thickness), and on L-MRIs, 8 patients showed decreases in thickness. We speculated that these phenomena might be correlated with the processes that gave rise to the spinal cord signal-intensity abnormalities. We also hypothesized that increased cord thickness on I-MRIs might, in part, be related to the resorption of posterior extradural fluid collections and the consequent resolution of compression on the dural sac and spinal cord.

One final reflection on changes in spinal cord thickness after surgery merits our attention. On E-MRIs, spinal cord thickness at the level of the herniation always seemed increased (re-expanded) compared with preoperative images. This finding seemed to be related to evidence of immediate clinical benefit after the operation. However, it was not possible to assess this finding accurately because when the spinal cord was herniated through the dura mater, its deformation made it difficult to measure cord thickness correctly.

Spinal cord contrast enhancement was not detected in our population. This was an expected result because it rarely occurs during follow-up in patients with chronic degenerative processes of the spinal cord. ${ }^{19}$ However, in our population, the administration of gadolinium showed dural patch contrast enhancement in 1 patient on the I-MRI (Fig 2). This finding may have indicated granulation tissue surrounding the patch, similar to scar tissue, which typically enhances in patients who have undergone prior a disc operation. $^{20}$

Nine patients underwent duraplasty; among these, we detected the dural patch on the E-MRIs in 3 cases, and on I-MRIs

Postoperative L-MRI findings in patients with new onset of neurologic symptoms

\begin{tabular}{|c|c|c|c|c|}
\hline $\begin{array}{l}\text { Patient } \\
\text { No. }\end{array}$ & $\begin{array}{l}\text { Anterior } \\
\text { Fluid } \\
\text { Collection }\end{array}$ & $\begin{array}{c}\text { Spinal } \\
\text { Superficial } \\
\text { Siderosis }\end{array}$ & $\begin{array}{c}\text { Brain } \\
\text { Superficial } \\
\text { Siderosis }\end{array}$ & Neurologic Symptoms \\
\hline 2 & Yes & No & Yes & $\begin{array}{l}\text { Persistent headache (worse in an upright } \\
\text { position), progressive SNHL }\end{array}$ \\
\hline 5 & Yes & Yes & Yes & $\begin{array}{l}\text { Persistent headache (worse in an upright } \\
\text { position), progressive SNHL }\end{array}$ \\
\hline 10 & Yes & No & Yes & Low back pain \\
\hline
\end{tabular}

Note:- SNHL indicates sensorineural hearing loss.
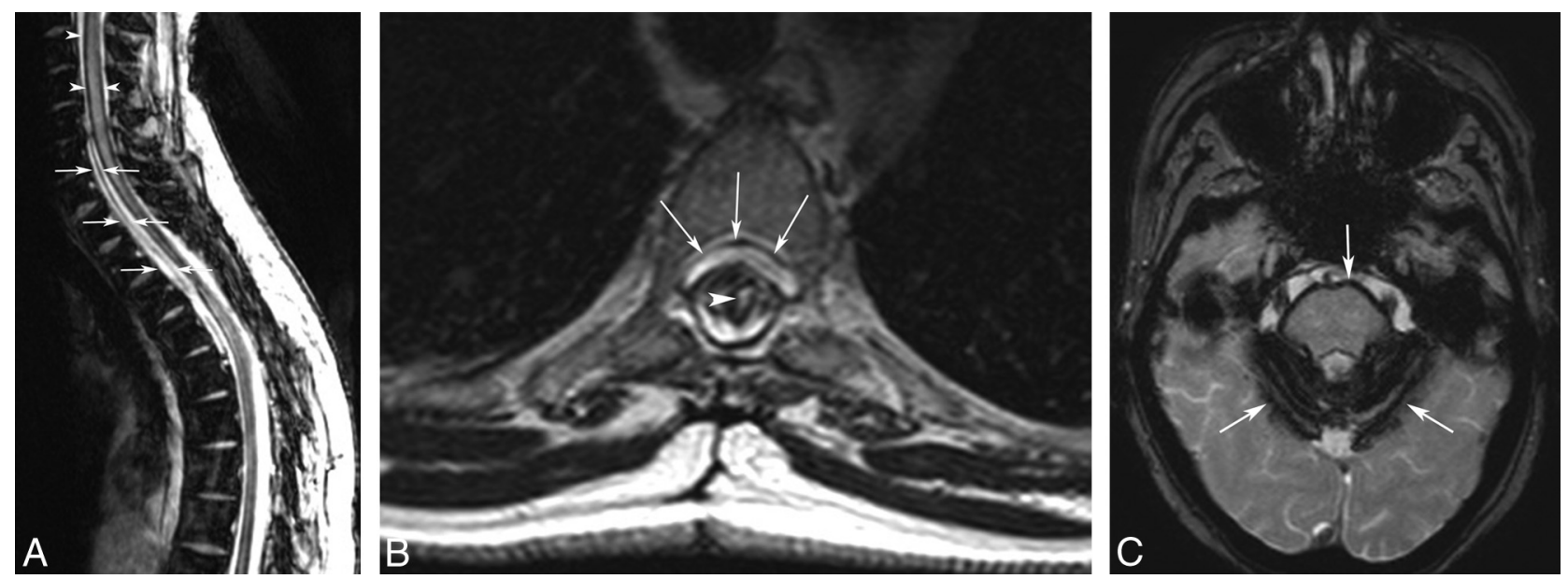

FIG 3. Anterior fluid collection and spinal and brain siderosis that developed 10 years after the operation for STSCH. A, Sagittal T2*WI demonstrates hemosiderin deposition along the spinal cord (arrowheads) and a cervicothoracic, anterior extradural fluid collection (arrows). $B$, Axial T2WI confirms the anterior fluid collection (arrows) and shows an intramedullary hyperintensity (arrowhead) and reduced spinal cord thickness at the level of the operation (D3-D4 vertebral bodies). C, Axial T2* of the brain demonstrates superficial siderosis (arrows). 
tients with STSCH treated with DDE; however, those patients lacked symptomatology related to a persistent CSF leak. Superficial siderosis is thought to result from recurrent subarachnoid bleeds, though the source of bleeding is not typically identified with imaging. ${ }^{22}$ The precise mechanism of bleeding in our cases remained unknown, but dural defects that were not repaired with duraplasty were probably the source of chronic or recurrent microhemorrhages. In fact, in a few reports of superficial siderosis, when a dural defect was detected, surgical repair of the dural tear with duraplasty stopped the bleeding. ${ }^{23}$ Moreover, a recent study described "duropathies" as conditions, like craniospinal hypovolemia, multisegmental amyotrophy, and STSCH, in which the common denominator was the presence of a dural defect with an intraspinal fluid collection, accompanied by superficial siderosis. ${ }^{24}$ To our knowledge, our 3 cases represent the first descriptions of superficial siderosis, which might indicate a long-standing complication of STSCH surgery.

The possible correlation between the duration of follow-up and the late MR imaging findings of fluid collection and siderosis deserves some consideration. L-MRI showed anterior fluid collection and siderosis from 2 to 10 years and between 8 and 17 years after surgery, respectively. These findings were observed in patients who had undergone DDE before the surgical technique was replaced by duraplasty. Due to the small sample of patients, we cannot conclude whether these MR imaging findings are related to the longer duration of follow-up and/or to the type of operation because in our population, the 2 factors coincided. Further studies with larger populations are needed to evaluate the possibility that these findings may actually correlate with longer follow-up duration, the type of operation, or both. Regarding spinal and/or intracranial pial siderosis, gradient-echo $\mathrm{T} 2{ }^{*}$, which represents the optimal sequence to detect spinal siderosis, was performed in only $8 \mathrm{~L}$-MRI examinations and in no preoperative MR imaging studies. No preoperative brain MR imaging studies were available. Thus, the evidence of spinal siderosis was based only on FSE T2WI for all preoperative studies and for most of the E-MRI and I-MRI studies. However, FSE can show pial siderosis, most likely in advanced stages, when the siderosis is extended and thick and the paramagnetic effect is marked, as reported by Boncoraglio et al, ${ }^{24}$ who described presurgical siderosis in a patient with spinal cord herniation.

Our study had some limitations. First and foremost, our study population was small, particularly when the different types of surgical techniques are considered separately (duraplasty and DDE). A separate analysis that compares postoperative MR imaging findings and clinical outcome in patients treated with DDE or duraplasty would have been interesting, to better understand the nature of the relationship between pial siderosis, extradural anterior fluid collection, and surgical procedures for STSCH and to determine the most appropriate surgical technique for STSCH. However, our small sample size precluded any meaningful statistical analyses. Another limitation was that our study included patients treated at a single institution; therefore, our results might not be generalizable to other neurosurgical units. To determine the effectiveness of surgical interventions for STSCH, we encourage other groups to perform follow-up studies that include de- tailed, comprehensive descriptions of postoperative MR imaging findings.

\section{CONCLUSIONS}

In this study, we demonstrated that a systematic, rational analysis of postoperative MR images acquired during the follow-up after STSCH treatment allowed detection of a wide spectrum of MR imaging changes that involved the spinal cord, intradural space, dura mater, and epidural space. An understanding of these postoperative changes and their evolution with time will provide the radiologist with the means to recognize the normal postoperative appearance of the spine and adjacent structures and to identify changes that may represent early and late postoperational complications.

Disclosures: Cesare Colosimo-UNRELATED: Consultancy: Bayer HealthCare, Bracco Diagnostics.

\section{REFERENCES}

1. Maira G, Denaro L, Doglietto L, et al. Idiopathic spinal cord herniation: diagnostic, surgical, and follow-up data obtained in five cases. J Neurosurg Spine 2006;4:10-19 CrossRef Medline

2. Parmar H, Park P, Brahma B, et al. Imaging of idiopathic spinal cord herniation. Radiographics 2008;28:511-18 CrossRef Medline

3. Sasani M, Ozer AF, Vural M, et al. Idiopathic spinal cord herniation: case report and review of the literature. J Spinal Cord Med 2009;32: 86-94 Medline

4. Imagama S, Matsuyama $Y$, Sakai $Y$, et al. Image classification of idiopathic spinal cord herniation based on symptom severity and surgical outcome: a multicenter study. J Neurosurg Spine 2009;11: 310-19 CrossRef Medline

5. Barrenechea IJ, Lesser JB, Gidekel AL, et al. Diagnosis and treatment of spinal cord herniation: a combined experience. J Neurosurg Spine 2006;5:294-302 CrossRef Medline

6. Haber MD, Nguyen DD, Li S. Differentiation of idiopathic spinal cord herniation from CSF-isointense intraspinal extramedullary lesions displacing the cord. Radiographics 2014;34:313-29 CrossRef Medline

7. Watanabe M, Chiba K, Matsumoto M, et al. Surgical management of idiopathic spinal cord herniation: a review of nine cases treated by the enlargement of the dural defect. J Neurosurg 2001;95(2 suppl): 169-72 Medline

8. Anmar KN, Pritchard PR, Matz PJ, et al. Spontaneous thoracic spinal cord herniation: three cases with long-term follow up. Neurosurgery 2005;57:E1067 CrossRef Medline

9. Nakamura M, Fujiyoshi K, Tsuji O, et al. Long-term surgical outcomes of idiopathic spinal cord herniation. J Orthop Sci 2011;16: 347-51 CrossRef Medline

10. Prada F, Saladino A, Giombini S, et al. Spinal cord herniation: management and outcome in a series of 12 consecutives patients and review of the literature. Acta Neurochir (Wien) 2012;154:723-30 CrossRef Medline

11. Kato S, Oshima Y, Oka H, et al. Comparison of the Japanese Orthopaedic Association (JOA) score and modified JOA (mJOA) score for the assessment of cervical myelopathy: a multicenter observational study. PLoS One 2015;10:e123022 CrossRef Medline

12. Aizawa T, Sato T, Sasaki H, et al. Results of surgical treatment for thoracic myelopathy: minimum 2-year follow-up study in 132 patients. J Neurosurg Spine 2007;7:13-20 CrossRef Medline

13. Colosimo C, Cianfoni A, Di Lella GM, et al. Contrast-enhanced MR imaging of the spine: when, why and how? How to optimize contrast protocols in MR imaging of the spine. Neuroradiology 2006; 48(suppl 1):18-33 CrossRef Medline

14. Grossman RI, Yousem DM. Neuroradiology: The Requisites. St Louis: Mosby; 1994:751-93

AJNR Am J Neuroradiol 37:558-64 Mar 2016 www.ajnr.org 
15. Groen RJ, Middel B, Meilof JF, et al. Operative treatment of anterior thoracic spinal cord herniation: three new cases and an individual patient data meta-analysis of $\mathbf{1 2 6}$ case reports. Neurosurgery 2009; 64(3 suppl):ons145-59; discussion ons159-60 CrossRef Medline

16. Chen CJ, Lyu RK, Lee ST, et al. Intramedullary high signal intensity on T2-weighted MR images in cervical spondylotic myelopathy: prediction of prognosis with type of intensity. Radiology 2001;221: 789-94 CrossRef Medline

17. Yagi $M$, Ninomiya $K$, Kihara $M$, et al. Long-term surgical outcome and risk factors in patients with cervical myelopathy and a change in signal intensity of intramedullary spinal cord on magnetic resonance imaging. J Neurosurg Spine 2010;12:59-65 CrossRef Medline

18. Sarkar S, Turel MK, Jacob KS, et al. The evolution of T2-weighted intramedullary signal changes following ventral decompressive surgery for cervical spondylotic myelopathy: clinical article. J Neurosurg Spine 2014;21:538-46 CrossRef Medline
19. Cho YE, Shin JJ, Kim KS, et al. The relevance of intramedullary high signal intensity and gadolinium (Gd-DTPA) enhancement to the clinical outcome in cervical compressive myelopathy. Eur Spine J 2011;20:2267-74 CrossRef Medline

20. Salgado R, Van Goethem JW, van den Hauwe L, et al. Imaging of the postoperative spine. Semin Roentgenol 2006;41:312-26 CrossRef Medline

21. Kumar N. Beyond superficial siderosis: introducing "duropathies." Neurology 2012;78:1992-99 CrossRef Medline

22. Kumar N, Cohen-Gadol AA, Wright RA, et al. Superficial siderosis. Neurology 2006;66:1144-52 CrossRef Medline

23. Kumar N. Neuroimaging in superficial siderosis: an in-depth look. AJNR Am J Neuroradiol 2010;31:5-14 CrossRef Medline

24. Boncoraglio GB, Ballabio E, Erbetta A, et al. Superficial siderosis due to dural defect with thoracic spinal cord herniation. J Neurol Sci 2012;312:170-72 CrossRef Medline 